\title{
Instrumental Effects in a Retarding Field Energy Analyzer*
}

\author{
Pax S. P. Weif and Aron Kuppermann \\ Arthur Amos Noyes Laboratory of Chemical Physics, $\ddagger$ California Institute of Technology, Pasadena, California 91109
}

(Received 25 October 1968; and in final form, 6 January 1969)

\begin{abstract}
Measurements of the well known electron impact spectra of helium show that a retarding field energy analyzer can give spurious responses; these can be minimized but not eliminated by careful adjustments of the operating potentials. Probable sources of the trouble are discussed.
\end{abstract}

$\mathrm{R}$ ETARDING field energy analyzers (RFEA) have been widely used in experiments to measure the energy distribution of electrons. It is well known that an RFEA is effective only in analyzing the momentum in a direction perpendicular to the equipotential lines. ${ }^{1}$ Ideally, we would like to have retarding grids made of $100 \%$ transparent fine mesh which could generate a well defined potential barrier. However, the finite size of the grid wires and the effect of field penetration give rise to practical difficulties.

In Penning ionization work ${ }^{2}$ and photoionization experiments, ${ }^{3}$ both having a cylindrically symmetrical scattering geometry, electron energy distribution spectra with resolution of $0.5 \mathrm{eV}$ or less have been reported. For the case of photoionization studies by a spherical RFEA with the ionization region located at its center, resolution to $0.01 \mathrm{eV}$ has been achieved. ${ }^{4}$ We have adopted a cylindrical scattering geometry similar to that described by Cermák ${ }^{2}$ and by Blake and Carver ${ }^{3}$ to study the electron impact spectra of gaseous samples with 30 to $50 \mathrm{eV}$ beams. Our results indicate that some unusual instrumental effects may contribute to the appearance of a spectrum and that it is important to understand and minimize these effects in order to interpret the information obtained with the RFEA. That these effects occur in electron impact but not in Penning or photoionization experiments can be attributed to the following reasons: (a) Metastable particles and photons are not subject to field penetration. (b) The electrons produced in the ionization experiments, ${ }^{2-4}$ being fewer and having less than $10 \mathrm{eV}$ energy, are less apt to produce secondary electrons from grids.

The purpose of this paper is to investigate the characteristics of a retarding field energy analyzer through the studies of the well known electron impact spectra of helium gas. Similar results associated with the electron impact spectra of a solid sample using a low energy electron diffraction apparatus will be discussed in a separate paper. ${ }^{5}$

\footnotetext{
* Work supported in part by the U.S. Atomic Energy Commission, Rep. Code No. CALT-532-34.

$f$ Present address: Bell Telephone Laboratories, Murray Hill, New Jersey 07971 .

$\ddagger$ Contribution No. 3730

1 . A. Simpson, Rev. Sci. Instrum. 32, 1283 (1961).

2 V. Cermák, J. Chem. Phys. 44, 3774, 3781 (1966).

${ }^{3}$ A. J. Blake and J. H. Carver, J. Chem. Phys. 47, 1038 (1967).

4 D. C. Frost, C. A. McDowell, and D. A. Vroom, Phys, Rev. Lett. $15,612(1965)$

5 P. S. P. Wei, A. Y. Cho, and C. W. Caldwell (unpublished).
}

The apparatus used in the present work is a modification of that described by Kuppermann and Raff. ${ }^{6,7}$ The only major improvement is the introduction of a Lozier-type grid $^{8}$ as G1 into the scattering chamber. This grid selects electrons scattered at $90^{\circ} \pm 6^{\circ}$, which sharpens the energy resolution when using an RFEA. Figure 1 shows a cross section of the cylindrically symmetrical scattering chamber grids and the electron gun, with the associated circuitry. The electron beam is directed into the scattering chamber through electrode E5 and is collected at the beam collector BC. E1, E2, E3, and E4 are focusing electrodes. The incident beam energy is determined by the potential on E5 which separates the gun from the scattering chamber and allows differential pumping between these two chambers. The potentials on G1 and G1A are kept equal to that on $\mathbf{E} 5$ to provide a field-free scattering region. The

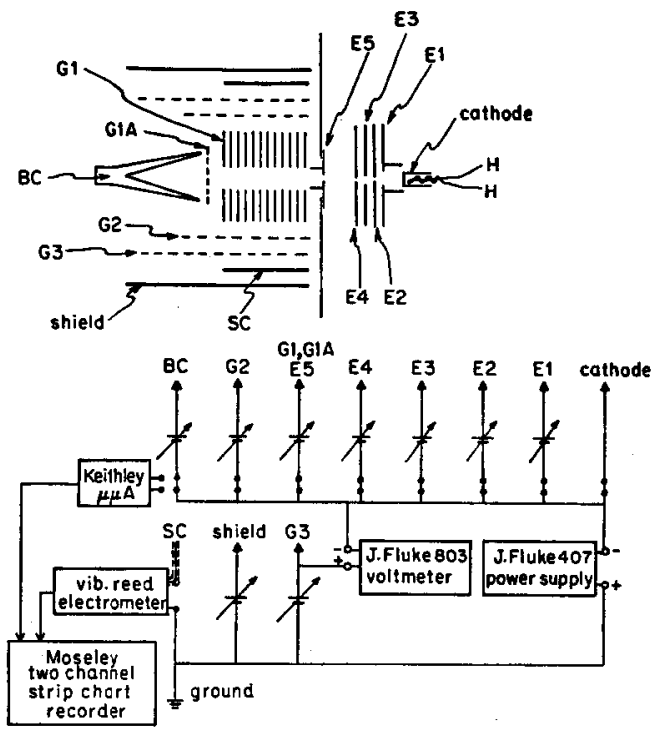

FIg. 1. Cross section of the cylindrically symmetrical scattering chamber grid system and the electron gun, with the associated circuitry. The set of potentials with respect to the cathode used for all experiments is as follows (unless otherwise stated): $\mathbf{E} 1=10 \mathrm{~V}$, $\mathrm{E} 2=4.5 \mathrm{~V}, \mathrm{E} 3=29 \mathrm{~V}, \mathrm{E} 4=6.5 \mathrm{~V}, \mathrm{E} 5=\mathrm{G} 1=\mathrm{G} 1 \mathrm{~A}=$ incident beam energy, $\mathrm{G} 2=\mathrm{E} 5+10 \mathrm{~V}, \mathrm{BC}=\mathrm{E} 5+70 \mathrm{~V}, \mathrm{G} 3=0$ to $30 \mathrm{~V}$, and $V_{\mathrm{sC} \text { to } \mathrm{Gz}}=V_{\text {shield to }} \mathrm{BC}=65 \mathrm{~V}-\mathrm{E} 5$. A spectrum is obtained by plotting the ratio of current increment at SC to the voltage increment at $\mathrm{G} 3$ vs $\mathrm{V}_{\mathrm{G} 3}$.

${ }^{6}$ A. Kuppermann and L. M. Raff, J. Chem. Phys. 37, 2497 (1962).

${ }^{7}$ A. Kuppermann and L. M. Raff, Discussions Faraday Soc. 35, 30 (1963).

${ }_{8}$ W. W. Lozier, Phys. Rev. 44, 575 (1935). 


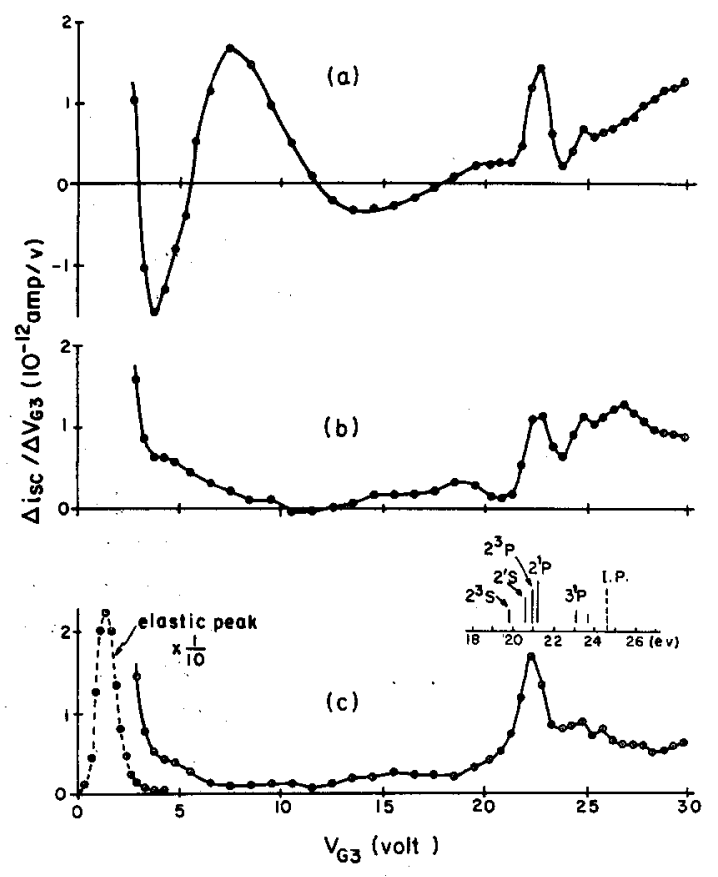

FIG. 2. Undulating background in $50 \mathrm{eV}$ helium spectra with $V_{\mathrm{SC}}$ to G3 at (a) 5 , (b) 10 , and (c) $15 \mathrm{~V}$. Beam current $=4.7 \times 10^{-7} \mathrm{~A}$, pressure $=1.5 \times 10^{-4}$ Torr (uncalibrated). The inserts in Figs. 2(c) and 3 represent the locations of the atomic energy levels of helium when the elastic peak is taken as zero of the energy loss scale.

potential on $\mathrm{G} 2$ is set at $10 \mathrm{~V}$ positive with respect to that on $\mathrm{E} 5$ to prevent positive ions from reaching the scattering collector SC. The holding potential between SC and G3 is determined by the experimental condition of yielding a minimum background. The energy loss of the scattered electrons is analyzed by sweeping the potential on G3 with respect to the cathode. The current increment on SC per unit retarding voltage is plotted vs the retarding potential $V_{\mathrm{G} 3}$ to give a spectrum.

Figure 2 shows three $50 \mathrm{eV}$ helium spectra with the holding potential between SC and G3 set at 5, 10, and $15 \mathrm{~V}$. The elastic peak is plotted at one-tenth of its height in Fig. 2(c). Since the lowest electronically excited state of helium is the $2^{3} S$ state at $19.81 \mathrm{eV}$ above the ground state, there should be a flat background between the elastic peak and the first energy loss peak. However, we see from these $50 \mathrm{eV}$ spectra that when the holding potential $V_{\mathrm{SC}}$ to $\mathrm{G}_{3}$ is set below $15 \mathrm{~V}$ there is an undulating background superimposed on the inelastic peaks. Similar tests for 40, 35, and $30 \mathrm{eV}$ electron beams indicate that the background undulation is smaller than $10 \%$ of the $21 \mathrm{eV}$ energy loss peak of helium whenever $V_{\mathrm{SC} \text { to G3 }}+V_{\text {incident }} \geq 65 \mathrm{~V}$. We also find that the full width at half-maximum (FWHM) of the elastic peak, which represents the resolution of our instrument, increases to $1.1 \mathrm{eV}$ with increasing $V_{\mathrm{SC}}$ to $\mathrm{G} 3$ when $\left(V_{\mathrm{SC}}\right.$ to $\left.\mathrm{G}_{3}+V_{\text {incident }}\right)=65 \mathrm{~V}$. Thus, impact spectra of molecules are taken under this condition for minimum background and moderate resolution. It is worthwhile to point out that the $50 \mathrm{eV}$ helium spectrum in Fig. 2(c) agrees very well with the $60 \mathrm{eV}$ helium spectrum reported by Doering. ${ }^{9}$

Figure 3 shows a $30 \mathrm{eV}$ helium spectrum with the inelastic structure superimposed on a rising background which exists as a large broad peak in the region where the residual energy of an electron is smaller than about $15 \mathrm{eV}$. This background moves away from the $20 \mathrm{eV}$ energy loss region when the incident beam energy is increased. Furthermore, due to the fact that G2 is set at $\mathrm{E} 5+10 \mathrm{~V}$, there is a second large broad background peak located $10 \mathrm{~V}$ higher than the one corresponding to the incident beam energy in the energy loss spectrum (not shown in Fig. 3). The dashed curve in Fig. 3 represents a blank run after shutoff of helium. It is noted that the rising background cannot be simply related to either the pressure or the elastic peak.

Figure 4 shows the $90^{\circ}$ electron impact spectra of helium with beam energies of $50,40,35$, and $30 \mathrm{eV}$. The most prominent feature of the spectrum is the unresolved $2^{1} P$ and $2^{3} P$ peak with a shoulder due to the $2^{1} S$ excitation. The $2^{3} S$ excitation is detected only at beam energies of 35 and $30 \mathrm{eV}$ in the present work. Our results (except the rising background) are consistent with the electron impact studies on helium by several authors. ${ }^{9-15}$

The present experiments on helium gas serve the important purpose of testing the instrument. We see that the undulating background may be minimized by using a suitably large holding potential between SC and G3. The rising background problem is most serious when we want to scan the energy loss region within about $15 \mathrm{eV}$ of the primary beam energy. Fortunately, the ionization potentials for many molecules are around $15 \mathrm{eV}$ or lower. Thus, we can still use the present technique to obtain electron impact spectra for those molecules at incident beam energies of $30 \mathrm{eV}$ or higher. Results obtained from several molecules will be presented elsewhere.

Concerning the fundamental sources of the trouble we believe that the following are most responsible.

(a) Field penetration. When measuring the scattered current at large energy loss, we have to set $V_{\mathrm{G} 3}$ at more and more positive potentials with respect to the cathode. The closer $V_{\mathrm{G} 3}$ gets to the value of $V_{\mathrm{G} 1}$, the greater is the field penetration into the electron beam region, with

9 J. P. Doering, J. Chem. Phys. 45, 1065 (1966).

${ }^{10}$ J. K. Rice, A. Kuppermann, and S. Trajmar, J. Chem. Phys. 48, 945 (1968).

11 J. A. Simpson, M. G. Menendez, and S. R. Mielczarek, Phys. Rev. 150, 75 (1966).

${ }^{12}$ G. E. Chamberlain, H. G. M. Heideman, J. A. Simpson, and C. E. Kuyatt, Proceedings of the International Conference on the Physics of Electronic and Atomic Collisions, $4 t h$, Book of Abstracts (Science Bookcrafters, Inc., New York, 1965), pp. 378-381.

${ }^{13}$ E. N. Lassettre, A. Skerbele, M. A. Dillon, and K. J. Ross, J. Chem. Phys. 48, 5066 (1968).

${ }_{14}$ J. P. Doering and A. J. Williams, III, J. Chem. Phys. 47, 4182 (1967).

${ }^{15}$ H. Ehrhardt and K. Willmann, Z. Physik 203, 1 (1967). 


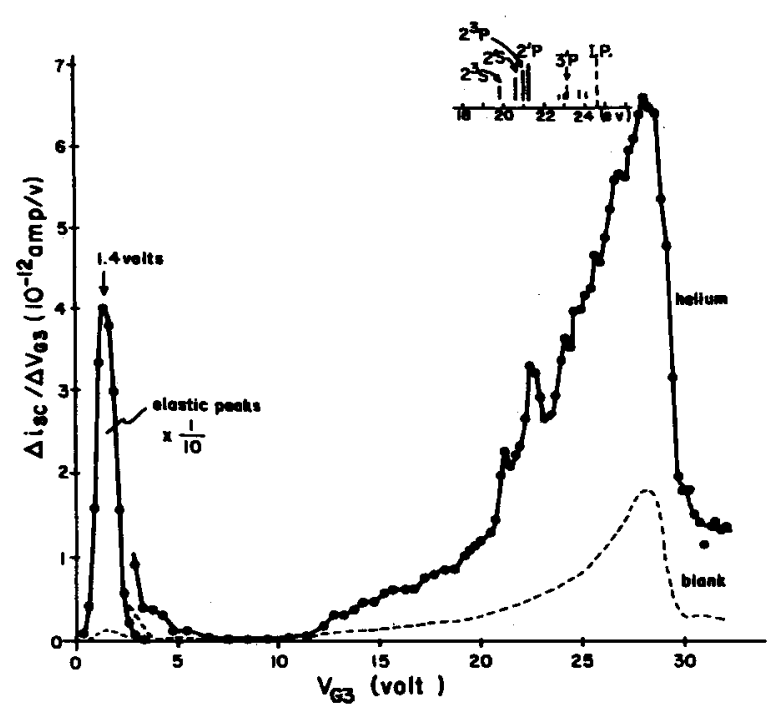

FIG. 3. Rising background in a $30 \mathrm{eV}$ helium spectrum with beam current $=0.98 \times 10^{-7} \mathrm{~A}$ and chamber pressure $=3.2 \times 10^{-4}$ Torr. The dashed curve is a blank run after shutoff of helium; beam current $=1.04 \times 10^{-7} \mathrm{~A}$ and pressure $=1.4 \times 10^{-5}$ Torr. It is obvious that the background is not simply related to either the pressure or the elastic peak.

consequent withdrawal of beam electrons which then reach $\mathrm{SC}$ without having been scattered.

(b) Scattering from grids. Elastic and inelastic scattering and secondary emission from the grids are unavoidable although all the grids and collectors are coated with platinum-black. Reflection and secondary emission from surfaces due to electron bombardment have been well known. ${ }^{16}$

(c) Insulating films. The phenomena of insulating films formed from organic substances deposited on surfaces under electron impact have been reported by several authors. ${ }^{17-19}$ Our diffusion pumps with Octoil-S pump oil and liquid nitrogen traps yield an ultimate vacuum of $\sim 5 \times 10^{-7}$ Torr in the system. However, the possiblilty of insulating films occurring on the grids and collectors after prolonged operation cannot be excluded.

It seems to us that the insulating films may be the major cause for the undulating background whereas field penetration and scattering from grids may account for the rising background.

${ }^{16}$ H. S. W. Massey and E. H. S. Burhop, Electronic and Ionic Impact Phenomena (Clarendon Press, Oxford, 1952), Chap. 5.

${ }^{17}$ R. L. Stewart, Phys. Rev. 45, 488 (1934).

${ }^{18}$ A. E. Ennos, Brit. J. Appl. Phys. 5, 27 (1954).

${ }^{10}$ R. E. Fox, W. M. Hickam, D. J. Grove, and T. Kjeldaas, Jr., Rev. Sci. Instrum. 26, 1101 (1955).

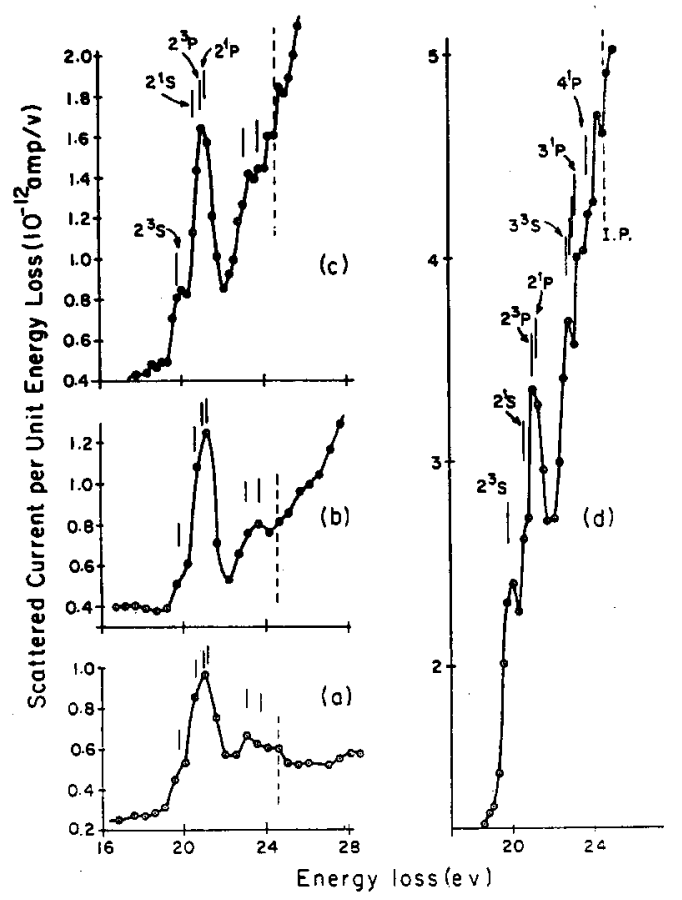

Frg. $4.90^{\circ}$ electron impact spectra of helium at beam energies of (a) 50, (b) 40, (c) 35, and (d) $30 \mathrm{eV}$. Beam current and pressure are the same as in Fig. 3. The spectra are plotted against the energy loss after contact potential corrections.

It is noted that Doering ${ }^{20}$ also observed a similar rising background in the impact spectrum of ethylene when he used a retarding field method to select the scattered electrons which then entered a $127^{\circ}$ cylindrical electrostatic analyzer. Recently, Doering and Williams ${ }^{14}$ were successful in eliminating this (what they called an electron-optical effect) by adding an electrostatic lens system between the scattering center and the $127^{\circ}$ analyzer.

Two remedial steps are suggested by the present experience, namely: (a) to test the instrument with a sample whose impact spectra are well established, then to perform experiments under allowable conditions; and (b) to replace the retarding field method by an electrostatic analyzer with well designed lens systems such as Kuyatt and Simpson's design. ${ }^{21}$ The latter step has been taken by Doering and Williams. ${ }^{14}$

We conclude that the instrumental effects discussed above are specific characteristics associated with the RFEA in electron impact work. We hope that the present work may serve as a reference for those who use similar techniques.

${ }^{20}$ J. P. Doering, J. Chem. Phys. 46, 1194 (1967).

${ }^{21}$ C. E. Kuyatt and J. A. Simpson, Rev. Sci. Instrum. 38, 103 (1967) 